\title{
A IMPORTÂNCIA DA IDENTIFICAÇÃO DE FATORES PSICOSSOCIAIS NA DOR LOMBAR CRÔNICA
}

\author{
THE IMPORTANCE OF IDENTIFICATION OF PSYCHOSOCIAL FACTORS IN \\ CHRONIC LOW BACK PAIN
}

\author{
Giselle Fernandes da Cunha \\ Acadêmica do 9º período de Fisioterapia na Faculdade Presidente Antônio Carlos/MG \\ E-mail: gisellecunha08@gmail.com \\ Letícia Santos Barbosa Matos \\ Acadêmica do 9º período de Fisioterapia na Faculdade Presidente Antônio Carlos/MG \\ E-mail: leticiamatos_21@hotmail.com \\ Ruana Santos Damascena \\ Acadêmica do 9º período de Fisioterapia na Faculdade Presidente Antônio Carlos/MG \\ E-mail: rdamascena4@gmail.com \\ André Luiz Velano de Souza \\ Bacharel em Fisioterapia pela PUC-MG (2003); Pós-graduado em Fisiologia do Exercício \\ pela Universidade Veiga de Almeida-RJ (2005) e Reabilitação Musculoesquelética e \\ Desportiva pela Universidade Gama Filho-RJ (2008) \\ E-mail: andrevelanofisio@gmail.com
}

Recebido: 07/06/2021 - Aceito: 20/06/2021

\begin{abstract}
Resumo
A dor se trata de um mecanismo de proteção existente no corpo humano que transmite sinais de alerta indicando que algo indesejável está sucedendo, o que nem sempre indica uma lesão e tampouco a causa da dor existente. Dor lombar é um importante problema de saúde pública que afeta cerca de $84 \%$ da população em algum momento da vida, sendo responsável por grandes índices de incapacidade e falta no trabalho, além de gerar altos custos para a sociedade, e pode ser conceituada como crônica quando tem duração maior que três meses. $O$ presente estudo trata-se de uma revisão de literatura, na qual foram consultados artigos publicados nas bases de dados virtuais: PEDro, Scielo e Pubmed; além de dados em sites oficiais renomados, com o objetivo de demonstrar a influência de fatores psicossociais no desenvolvimento e persistência da dor lombar crônica, de forma a possibilitar a integração de um tratamento adequado na prática fisioterapêutica. Há um grande indício de que pacientes com dor lombar crônica tenham alguma alteração psicossocial acompanhada que colaboram para a sustentação do estado de dor e maior restrição às atividades, o que implica a necessidade de se atentar a esses fatores na avaliação. Com isso, a inclusão de questionários de avaliação psicossocial, como o Start Back Screenig Tool e o Orebro Musculoskeletal Pain Screening Questionnaire, são úteis na promoção e garantia de uma reabilitação mais eficiente, contribuindo na triagem dos pacientes quanto ao risco de um prognóstico ineficaz no tratamento primário.
\end{abstract}

Palavras-chave: dor lombar crônica; fatores psicossociais; avaliação; questionários; fisioterapia. 


\begin{abstract}
Pain is a protective mechanism existing in the human body that transmits warning signals indicating that something undesirable is happening, which does not always indicate an injury or the cause of the existing pain. Low back pain is an important public health problem that affects about $84 \%$ of the population at some point in their lives, being responsible for high rates of disability and absence from work, in addition to generating high costs for society, and can be conceptualized as chronic when it lasts longer than three months. This study is a literature review, in which articles published in the following virtual databases were consulted: PEDro, Scielo and Pubmed; in addition to data on renowned official websites, with the aim of demonstrating the influence of psychosocial factors on the development and persistence of chronic low back pain, in order to enable the integration of an adequate treatment in physical therapy practice. There is a strong indication that patients with chronic low back pain have some psychosocial alteration followed that collaborate to sustain the state of pain and greater restriction to activities, which implies the need to pay attention to these factors in the assessment. Thus, the inclusion of psychosocial assessment questionnaires, such as the Start Back Screenig Tool and the Orebro Musculoskeletal Pain Screening Questionnaire, are useful in promoting and ensuring a more efficient rehabilitation, contributing to the screening of patients for the risk of an ineffective prognosis in primary treatment.
\end{abstract}

Keywords: chronic low back pain; psychosocial factors; assessment; questionnaires; physiotherapy.

\title{
1. Introdução
}

A dor se trata de um mecanismo de proteção existente no corpo humano que envia sinais de alerta indicando que algo de errado está ocorrendo e nem sempre aponta uma lesão e tampouco a causa da dor existente. Além de se tratar de um fenômeno subjetivo e complexo, pode ser influenciado não apenas por fatores físicos, mas também genéticos, emocionais, cognitivos e memórias. As evidências atuais mostram que alguns fatores como a hipervigilância, pensamentos e crenças negativas, catastrofização da dor, expectativas em relação ao quadro, medo do movimento, ansiedade, estresse e depressão colaboram para a sustentação do estado de dor crônica, maior restrição às atividades e incapacidade (TEGNER et al., 2018).

Dor lombar é definida como dor ou desconforto localizado na região posterior do corpo, abaixo da margem costal e acima da linha glútea superior com ou sem dor irradiada para membro inferior (ALMEIDA; KRAYCHETE, 2017). 
Dellaroza et al. (2008) diz que a dor crônica pode ser conceituada como uma dor que dura mais de três meses, com a função de alertar o organismo que existe algo acontecendo de forma incorreta, e na maioria das vezes não tem uma causa definida, e além disso, não desaparece com a aplicação de técnicas terapêuticas convencionais, sendo também a razão de diversas incapacidades e inabilidades prolongadas.

De acordo com Nascimento e Costa (2015), dor lombar pode afetar até $84 \%$ das pessoas em algum momento da vida, até 65\% das pessoas anualmente e possui prevalência de $11,9 \%$ na população mundial, sendo causa de grande demanda nos serviços de saúde. No Brasil, os quadros crônicos de dor lombar atingem de $4,2 \%$ a $14,7 \%$ da população.

Segundo Hoy et al. (2012), é um problema de saúde muito comum, que pode aumentar nas próximas décadas devido ao envelhecimento da população, sendo responsável por grandes índices de incapacidade e falta no trabalho, além de gerar altos custos para a sociedade.

Em relação aos altos custos associados a dor nas costas, destacam-se os gastos com auxílios devido à afastamentos do trabalho, exames de imagem, cirurgias, etc. De acordo com o Instituto Nacional de Seguro Social (2020), a dorsalgia tem liderado a lista de doenças mais frequentes entre os auxílios-doença concedidos nos últimos dez anos, e foi a doença que mais afastou os brasileiros dos seus trabalhos em 2017, contabilizando 83,8 mil casos. Vale ressaltar que esses dados correspondem aos auxílios-doença previdenciários, ou seja, não possuem relação com acidente ou doença do trabalho, sendo que nessa categoria ocupou o terceiro lugar em 2016 e o quinto lugar em 2017, com 11,8 mil auxílios.

Os custos com cirurgias também são muito expressivos, uma vez que houve um aumento de $226 \%$ no número e $540 \%$ no custo total das cirurgias de coluna no 
sistema público de saúde brasileiro nos últimos 20 anos (TELES et al., 2016).

Outro fator responsável pelos altos custos relacionados com problemas da coluna vertebral é a crescente utilização de exames de imagem. Em 2016, apenas no sistema público de saúde foram gastos mais de 13 milhões de dólares em diagnóstico por imagem durante o ambulatório e internação, sendo as maiores despesas com ressonância magnética e tomografia computadorizada (CARREGARO; DA SILVA; TULDER, 2019).

A ideia de que dor é sempre gerada por uma lesão leva muitos profissionais a considerar o exame de imagem como indispensável para a avaliação e tratamento dos pacientes, o que não é uma verdade absoluta, e nem se aplica a todos os quadros. Essa constante utilização desses exames de imagem aumenta os custos relacionados à dor lombar devido aos próprios procedimentos relacionados ao exame, além de gastos necessários para acompanhamento, encaminhamento e muitas vezes até em tratamentos invasivos limitados e questionáveis. Segundo Chou et al. (2011), as imagens de rotina não estão relacionadas a benefícios clinicamente significativos e expõe os pacientes com dor lombar à danos desnecessários, visto que as alterações detectadas são comuns em pacientes assintomáticos por serem sinais de envelhecimento e não significam necessariamente que são responsáveis pelos sintomas.

Pensando nisso, a pesquisa promoverá a difusão do tema esclarecendo os mecanismos básicos da dor correlacionados com os fatores psicossociais que interferem na permanência dessa condição, e com isso mostrar a importância da identificação e como pode ser feito o reconhecimento desses agentes. Os resultados poderão auxiliar os profissionais da saúde que atuam diretamente no tratamento de dor crônica, ajudando na redução de gastos econômicos relacionados a condutas e tratamentos, como por exemplo exames de imagem que 
nem sempre são capazes de evidenciar a origem da dor. Nesse sentido, o objetivo desse estudo foi demonstrar a influência dos fatores psicossociais no desenvolvimento e persistência da dor lombar crônica, de forma a possibilitar a integração de um tratamento adequado na prática fisioterapêutica.

Tendo isso em vista, além da importante influência dos fatores psicossociais na dor lombar crônica, percebe-se a necessidade de uma avaliação mais abrangente, que leve em consideração não apenas aspectos físicos, como também psicológicos e sociais.

O presente estudo trata-se de uma revisão de literatura, desenvolvida através de pesquisa bibliográfica de natureza qualitativa, a nível descritivo e exploratório, sendo realizada uma leitura analítica criteriosa de artigos publicados nas bases de dados virtuais: PEDro, Scielo e Pubmed; além de dados em sites oficiais renomados. Foram analisadas informações de diversas fontes para a construção, inclusive obras clássicas sobre o tema, priorizando publicações mais recentes, em língua portuguesa e inglesa. Trabalhos em outros formatos como monografias, dissertações e teses não foram consultados.

\section{Revisão Bibliográfica}

\subsection{Conceito de dor e suas características}

Devido às mudanças que aconteceram na compreensão do mecanismo da dor é entendido que nem sempre a mesma está relacionada a uma lesão tecidual evidente, e que o estado emocional do paciente afeta de modo direto na dor que ele sente. Com isso, pacientes com problemas que influenciam o controle psicológico podem apresentar quadros mais intensos de dor relatada. Consequentemente, foi compreendido que a concepção de dor se apresenta de 
maneira individual e com alta interferência de fatores psicossociais (SOUZA; BARROS, 2020).

A Associação Internacional para o Estudo da Dor (IASP) conceitua a dor como "uma experiência sensitiva e emocional desagradável associada, ou semelhante àquela associada, a uma lesão tecidual real ou potencial”. Essa definição é acompanhada por 6 notas explicativas, na qual a primeira nota diz que "a dor é sempre uma experiência pessoal que é influenciada, em graus variáveis, por fatores biológicos, psicológicos e sociais" (IASP, 2020).

De acordo com Dellaroza et al. (2008) a longa duração da dor crônica causa uma perda da capacidade de manter a homeostase e de exercer a função de sinal de alerta, gerando comprometimento da funcionalidade, sofrimento, incompetência progressiva e custo socioeconômico. É sabido que o acometimento da dor crônica, independente da patologia de base, apresenta consequências na saúde dos pacientes, fazendo com que essa condição receba a atenção dos profissionais de saúde.

Para Kreling, Cruz e Pimenta:

A dor aguda ou crônica, de um modo geral, leva o indivíduo a manifestar sintomas como alterações nos padrões de sono, apetite e libido, manifestações de irritabilidade, alterações de energia, diminuição da capacidade de concentração, restrições na capacidade para as atividades familiares, profissionais e sociais. Nos indivíduos com dor crônica, a persistência da dor prolonga a existência desses sintomas, podendo exacerbá-los (KRELING; CRUZ; PIMENTA, 2006, p. 509-510).

Segundo Dellaroza et al. (2008), mais de um terço da população brasileira acredita que a dor crônica implica nas atividades de vida diária e mais de três 
quartos julga que esta condição é restritiva para as atividades recreacionais, relações sociais e familiares.

\subsection{Influência dos Fatores Psicossociais no Desenvolvimento e Persistência da Dor Lombar Crônica}

Fatores emocionais e cognitivos como hipervigilância, catastrofização, ansiedade e depressão comumente influenciam a percepção da dor em indivíduos que apresentam dor crônica através de um sistema de modulação da dor, que afetam tanto na intensidade, como na persistência dessa dor (TEGNER et al., 2018).

De acordo com Linton e Shaw (2011), os principais fatores que podem interferir na experiência da dor são: a atenção, a cognição, as emoções e os comportamentos que estão descritos na seguinte Tabela.

Tabela: Principais fatores que interferem na experiência da dor

\begin{tabular}{|l|l|}
\hline Fator & Possível influência na dor e incapacidade \\
\hline Atenção & $\begin{array}{l}\text { A atenção é necessária para a percepção da dor e para } \\
\text { haver respostas adequadas em quadros de lesões. Sua } \\
\text { função psicológica é motivar o comportamento. Quando a } \\
\text { dor é entendida como uma "ameaça", quanto mais } \\
\text { "ameaçadora" for considerada mais atenção é dada a ela. } \\
\text { Sendo assim, ela pode estar relacionada ao medo, a } \\
\text { ansiedade e a necessidade de agir (por exemplo, fugir ou } \\
\text { evitá-la). Em situações em que essa atenção passa a ser } \\
\text { exacerbada, poderá resultar na hipervigilância, que é um } \\
\text { fator que tende a aumentar a intensidade da sensação da } \\
\text { dor. }\end{array}$ \\
\hline Cognição & $\begin{array}{l}\text { Os processos cognitivos atuam na interpretação do } \\
\text { "estímulo nocivo", por isso a forma que pensamos sobre a } \\
\text { nossa dor pode influenciá-la e as experiências anteriores } \\
\text { podem moldá-la. Dentro desse modelo destacam-se os }\end{array}$ \\
\hline
\end{tabular}




\begin{tabular}{|c|c|}
\hline & $\begin{array}{l}\text { pensamentos e crenças negativas, a catastrofização e as } \\
\text { expectativas, que podem aumentar a dor e a incapacidade. }\end{array}$ \\
\hline Emoções & $\begin{array}{l}\text { Sabe-se que a dor interfere nas emoções e comumente } \\
\text { gera sentimentos negativos como a angústia emocional, e } \\
\text { reações como ansiedade, medo, raiva, culpa, frustração e } \\
\text { até mesmo depressão. A forma como essas emoções são } \\
\text { reguladas pelo paciente tem implicações no quadro de dor e } \\
\text { em seus impactos. Esses sentimentos negativos podem } \\
\text { também estimular a cognições, a atenção e os } \\
\text { comportamentos. O medo, por exemplo, aumenta o } \\
\text { comportamento de evitação e deficiência. Já a ansiedade, a } \\
\text { depressão e a angústia aumentam a incapacidade e o } \\
\text { quadro álgico, e no caso da angústia há também a } \\
\text { possibilidade de estímulo de cognições negativas. Em } \\
\text { contrapartida, emoções positivas podem diminuir a dor. }\end{array}$ \\
\hline Comportamento & $\begin{array}{l}\text { A forma como lidamos com a dor também influencia na } \\
\text { percepção da mesma. Vale ressaltar que a maioria dos } \\
\text { comportamentos de dor são aprendidos e influenciados por } \\
\text { emoções, cognições, e por fatores ambientais e sociais. } \\
\text { Destaca-se que os comportamentos de evitação e de dor } \\
\text { podem piorar o quadro, gerando maior incapacidade. }\end{array}$ \\
\hline
\end{tabular}

Fonte: LINTON E SHAW (2011) (p. 702-704)

Para Main, Foster e Buchbinder (2010), a percepção da dor é uma consequência de sua interpretação, que por sua vez é moldada por nossas memórias e experiências anteriores. Já a resposta à dor é influenciada por crenças sobre ela e pelo significado emocional que atribuímos à mesma. Os autores apontam que as crenças sobre a natureza da dor, medo de gerar mais danos e lesões e as crenças de autoeficácia são as mais importantes a serem consideradas. Junto a isso, indicam que as influências sociais desempenham um papel importante na determinação do resultado da dorsalgia e no desenvolvimento de incapacidade. 
No estudo de Apeldoorn et al. (2012), realizado na Holanda com 229 pacientes com dor lombar crônica que frequentaram um centro ambulatorial, 53\% dos pacientes apresentaram alterações psicológicas consideráveis. Ainda segundo o estudo, fatores sociais contribuem para o desenvolvimento e persistência da dor lombar, devem ser analisados durante a avaliação e podem interferir no resultado do tratamento.

Em um estudo realizado por Dellaroza et al. (2008), com 172 idosos na cidade de Londrina em 2006, no qual o objetivo foi caracterizar a dor crônica e os métodos analgésicos utilizados pelos mesmos, foi possível analisar que 107 idosos apresentam dor crônica, no qual $92,52 \%$ são da classe social C, D ou E, 97,1\% apontam déficit cognitivo e 56,1\% apresentam depressão. Dessa forma, é notória a grande proporção de dor crônica acompanhada de fatores psicossociais, principalmente aqueles de média e baixa classe social e com déficit cognitivo.

\subsection{Relação da dor lombar inespecífica com achados radiológicos}

Segundo Hall et al. (2021), nas últimas décadas ocorreram mudanças em relação ao uso de exames de imagem para diagnóstico de dor lombar. Embora as diretrizes da American College of Physicians (ACP) e American Pain Society (APS) não indiquem exames de imagem como radiografia lombar, tomografia computadorizada, ressonância magnética e outras imagens avançadas para dor lombar inespecífica, esta abordagem é realizada frequentemente. A excessiva utilização de exames de imagem para avaliação é um dos principais contribuintes para os altos gastos com dor lombar e aproximadamente um terço desses exames são realizados de forma desnecessária. Conforme as recomendações da ACP e APS a realização de exames de imagem é útil apenas em pacientes que apresentam suspeita de patologias graves, que são casos raros, equivalente a 5$10 \%$ de todos os casos de dor lombar na atenção primária. Os outros $90-95 \%$ que 
não apresentam problemas graves devem ser tratados de maneira conservadora, pois submetê-los a exames de imagem pode causar mais danos do que benefícios. Uma avaliação criteriosa, coletando história clínica e exame físico poderá distinguir se o paciente apresenta dor lombar específica tendo como causa alguma patologia como câncer, infecção espinhal, doença inflamatória, fratura, síndrome da cauda equina e déficits neurológicos ou dor lombar inespecífica, em que a causa não pode ser associada a alguma alteração na estrutura anatômica.

O estudo de Jacobs et al. (2020) realizado com 405.965 pacientes da atenção primária dos EUA descobriu que aqueles que realizaram ressonância precocemente sem indicação tiveram maior probabilidade de submeter à cirurgia na coluna, fazer uso de opioides prescritos, custos mais elevados e após um ano de acompanhamento a pontuação de dor era maior em comparação com aqueles que não realizaram a abordagem precoce.

Em uma revisão de literatura realizada por Brinjikji et al. (2015), o objetivo do estudo foi determinar a prevalência específica para achados de imagem associados à doença degenerativa da coluna em pacientes assintomáticos. Os resultados da revisão sistemática mostram que degeneração discal estava presente em $37 \%$ dos exames de imagem de pacientes com 20 anos e $96 \%$ em pacientes com 80 anos, hérnia de disco foi observada em $30 \%$ dos exames de pacientes com 20 anos e $84 \%$ em pacientes de 80 anos, protusão discal em $29 \%$ dos pacientes com 20 anos e $43 \%$ em pacientes com 80 anos. Isso mostra que alterações de degeneração espinhal são comuns em pacientes assintomáticos, aumentam com a idade podendo ser vistas como sinais normais do envelhecimento e que nem sempre essas alterações são a causa da dor do paciente.

De acordo com Chou et al. (2011), a solicitação dos exames de imagem quando não possui indicação clara pode estar relacionada com o desejo e maior 
satisfação do paciente. Para alguns pacientes a solicitação de exames de imagem pode estar relacionada com a qualidade do atendimento e desejo de um diagnóstico preciso para sua dor. Apesar da percepção de que a solicitação do exame pode aliviar a ansiedade do paciente em relação à dor, essa abordagem não causa melhores resultados psicológicos, uma vez que se caso exista alterações estruturais, após receber o exame, a falta de conhecimento de que aquelas alterações podem ser normais e não possuir relação com a dor, irá causar ao paciente mais preocupação com pequenos sintomas na coluna lombar, fazendo com que pequenos exercícios e atividades que seriam benéficos sejam evitadas por medo de causar mais danos estruturais, levando ao retardamento do tratamento que de fato seria eficaz.

Reduzir exames desnecessários ou tratamentos ineficazes é fundamental para diminuir os custos com dor lombar, uma vez que não melhora resultados e expõem pacientes a danos desnecessários. Portanto, uma forma para solucionar o problema é educar o paciente para equiparar suas preferências com as evidências, informando-lhe sobre a eficácia e danos causados pelos procedimentos (CHOU et al., 2007).

\subsection{Avaliação}

O estudo de Oliveira et al. (2018) teve como objetivo fornecer uma visão geral das recomendações relativas aos diagnósticos e tratamentos contidas nas diretrizes de prática clínica atuais para pacientes com dor lombar inespecífica na atenção primária e verificar alterações das recomendações entre os anos de 2008 e 2017. De acordo com o estudo, quinze diretrizes de prática clínica foram publicadas ou atualizadas entre esse período. Houve algumas diferenças em relação à visão geral anterior, inclusive sobre as recomendações para avaliação de fatores psicossociais. Recomendações em relação ao diagnóstico de dor lombar 
foram encontradas em 13 estudos e os resultados mostram que mais da metade das diretrizes (54\%) recomendam triagem diagnóstica classificando o paciente em uma das três categorias: dor lombar inespecífica, dor lombar associada a radiculopatia ou estenose espinhal, ou dor lombar específica. A maioria das diretrizes (83\%) recomendam anamnese e exame físico para indicar presença de sinais graves, nenhuma recomenda a solicitação de exames de imagem de rotina, $58 \%$ sugerem que a imagem só deve ser solicitada se houver sinais de alerta e $17 \%$ se a dor persistir por mais de quatro a seis semanas. Doze diretrizes apresentaram recomendações para avaliação de fatores psicossociais para identificar pacientes quanto ao risco de mau prognóstico. Uma proporção crescente das diretrizes (33\%), recomendam a avaliação de fatores psicossociais através de ferramentas de triagem como os questionários StarTBack Screening Tool e Orebro Musculoskela Pain.

Diretrizes de prática clínica para dor lombar não específica destacam a importância de identificar na atenção primária, fatores que indicam mau prognóstico para melhor direcionamento do futuro tratamento (CHOU et al., 2007).

Instrumentos de triagem validados como StarTBack e Orebro podem ser utilizados para avaliar fatores psicossociais, facilitando a triagem de pacientes para a escolha do melhor tratamento (KEEFFE et al., 2018).

\subsubsection{StarTBack Screening Tool}

Hill et al. (2008) desenvolveram e validaram o questionário StarTBack Screening Tool na língua inglesa com o objetivo de classificar pacientes com dor lombar inespecífica quanto ao risco de mau prognóstico na atenção primária em relação à presença de fatores físicos e psicológicos. 
O estudo de Pilz et al. (2014) teve como objetivo traduzir e adaptar transculturalmente o questionário para a língua portuguesa do Brasil e testar sua confiabilidade. O questionário é composto por nove itens em que os quatro primeiros são relacionados à dor, incapacidade e comorbidades, já os demais itens estão associados às questões psicossociais. Os pacientes são classificados em alto risco de mau prognóstico quando apresentam alto nível de fatores psicossociais com ou sem presença de fatores físicos, médio risco de mau prognóstico pacientes que apresentam fatores físicos e psicossociais em níveis mais baixos que a classificação anterior e baixo risco de mau prognóstico quando os fatores físicos e psicossociais são mínimos. A tradução do questionário teve bons resultados similares ao questionário original, sendo uma ferramenta eficaz para ser utilizada na triagem de pacientes com dor lombar no Brasil.

No estudo de Hill et al. (2011) os autores verificaram que pacientes classificados de alto risco de mau prognóstico possuem presença de fatores físicos e psicossociais e devem receber tratamento baseado em uma abordagem física e psicológica, caso contrário, podem não evoluir adequadamente. Já os pacientes classificados como médio risco de mau prognóstico devem realizar tratamento fisioterapêutico para melhora de sintomas e função, além de orientações, caso necessário. Por outro lado, os pacientes classificados em baixo risco de mau prognóstico necessitam de mudanças em relação aos hábitos de vida, prática de atividades físicas e orientações em relação aos sintomas. No estudo, pacientes que foram classificados em baixo, médio e alto risco e realizaram tratamento como descrito acima tiveram redução do uso de serviços de saúde, melhora da função na escala do Questionário de Incapacidade de Roland e Morris, diminuição de afastamentos de trabalho e melhora da qualidade de vida em comparação aos pacientes que não se submeteram a aplicação do questionário na avaliação. 


\subsubsection{Orebro Musculoskeletal Pain Screening Questionnaire}

O estudo de Fagundes et al. (2015) teve o objetivo de traduzir, adaptar culturalmente e testar as propriedades de medida das versões curta e longa do Orebro Musculoskeletal Pain Screening Questionnaire na língua portuguesa do Brasil. A versão longa do questionário tem a finalidade de classificar pacientes com dor lombar aguda e subaguda não específica em baixo, médio e alto risco de desenvolver dor crônica e incapacidade associada a fatores psicossociais. Já a versão curta classifica os pacientes apenas em baixo e alto risco. As versões longa e curta avaliam os mesmos fatores: dor, função, cinesiofobia, variáveis psicológicas e variáveis relacionadas à demografia, ambiente e fatores relacionados ao trabalho. Os resultados do estudo mostram que as versões longa e curta apresentaram valores aceitáveis em todos os testes de propriedades de medidas e são semelhantes às versões originais.

O estudo de Linton e Koersma (2003) buscou avaliar a utilidade clínica do questionário Orebro em pacientes que afastaram do trabalho por licença médica e analisar a capacidade do questionário em prever resultados, incluindo dor, função e falta ao trabalho. Segundo o estudo, o questionário Orebro é uma ferramenta confiável e válida que pode ser utilizada como complemento na triagem de pacientes, para identificar de forma precoce o risco de desenvolver dores crônicas, sendo útil para prever possíveis incapacidades e falta ao trabalho devido à doença.

\section{Considerações Finais}

O desenvolvimento do presente estudo possibilitou uma análise da ação da dor, na qual se caracteriza como um mecanismo de proteção do organismo que conduz sinais de alerta, sendo evidente que nem sempre a mesma está relacionada a uma lesão e o estado emocional do paciente afeta de modo direto na sensação de dor, mostrando que a correlação de fatores como a hipervigilância, 
pensamentos e crenças negativas, emoções, medo do movimento (cinesiofobia), ansiedade, depressão e estresse contribuem para o prolongamento do estado de dor crônica e maior limitação às atividades.

Além disso, foi evidenciado que a degeneração da coluna se intensifica com o avanço da idade podendo se apresentar como sinal comum do envelhecimento e que nem sempre é a causa da dor do paciente. Nesse sentido, o alto índice de aplicação de exames de imagem realizados de maneira indevida para avaliação atinge o sistema de saúde em termos econômicos, pois se apresenta como um dos grandes contribuintes para os altos gastos com dor lombar.

Desse modo, verificar a existência de fatores psicossociais é crucial para averiguar riscos de um prognóstico ineficaz no tratamento primário. Com isso, a inclusão de questionários de avaliação psicossocial, como o Start Back e Orebro, são úteis na promoção e garantia de uma reabilitação mais eficiente, contribuindo na triagem dos pacientes. Vale ressaltar que é importante que o profissional tenha compreensão sobre cada ferramenta para determinar qual vai ser aplicada em cada paciente.

\section{Referências}

ALMEIDA, Darlan Castro; KRAYCHETE, Durval Campos. LOW BACK PAIN - A DIAGNOSTIC APPROACH. Revista Dor, São Paulo, Sp, v. 18, n. 2, p. 173-177, abr- jun. 2017. GN1 Genesis Network. http://dx.doi.org/10.5935/18060013.20170034.

https://www.scielo.br/j/rdor/a/9JxZrqLhB7r5y8rKWtXDYXt/?lang=en. Acesso em: 30 abr. 2021.

APELDOORN, A.T. et al. IDENTIFICATION OF PATIENTS WITH CHRONIC LOW BACK PAIN WHO MIGHT BENEFIT FROM ADDITIONAL PSYCHOLOGICAL ASSESSMENT. Clin J Pain, [S.L.], v.28, n. 1, jan. 2012 doi: 
10.1097/AJP.0b013e31822019d0.

em: https://journals.Iww.com/clinicalpain/Abstract/2012/01000/Identification_of_Patients _With_Chronic_Low_Back.4.aspx. Acesso em: 16 mai. 2021.

BRASIL. Ministério da Economia. Secretaria da Previdência. Saúde Do Trabalhador: Dor nas costas foi doença que mais afastou trabalhadores em 2017. Jun. 2020. Disponível em: https://www.gov.br/previdencia/ptbr/assuntos/noticias/previdencia/institucional/saude-do-trabalhador-dor-nas-costasfoi-doenca-que-mais-afastou-trabalhadores-em-2017. Acesso em: 16 mai. 2021.

BRINJIKJI, W. et al. SYSTEMATIC LITERATURE REVIEW OF IMAGING FEATURES OF SPINAL DEGENERATION IN ASYMPTOMATIC POPULATIONS. American Journal Of Neuroradiology, [S.L.], v. 36, n. 4, p. 811 816, 27 nov. 2014. American Society of Neuroradiology (ASNR). http://dx.doi.org/10.3174/ajnr.a4173. Disponível em: http://www.ajnr.org/content/36/4/811.long. Acesso em: 08 mar. 2021.

CARREGARO, Rodrigo Luiz; SILVA, Everton Nunes da; VAN TULDER, Maurits. DIRECT HEALTHCARE COSTS OF SPINAL DISORDERS IN BRAZIL. International Journal Of Public Health, [S.L.], v. 64, n. 6, p. 965-974, 15 maio 2019. Springer Science and Business Media LLC. http://dx.doi.org/10.1007/s00038019-01211-6. Disponível em: https://link.springer.com/article/10.1007\%2Fs00038019-01211-6. Acesso em: 08 mai. 2021.

CHOU, Roger et al. DIAGNOSIS AND TREATMENT OF LOW BACK PAIN: A JOINT CLINICAL PRACTICE GUIDELINE FROM THE AMERICAN COLLEGE OF PHYSICIANS AND THE AMERICAN PAIN SOCIETY. Annals of Internal Medicine, [S.L.], v. 147, n. 7, p. 478-491, 2 out. 2007. American College of Physicians. http://dx.doi.org/10.7326/0003-4819-147-7-200710020-00006. 
Revista Multidisciplinar do Nordeste Mineiro, v.2,

$2021 / 02$

ISSN 2178-6925

Disponível em: https://www.acpjournals.org/doi/pdf/10.7326/0003-4819-147-7200710020-00006. Acesso em: 16 abr. 2021.

CHOU, Roger et al. DIAGNOSTIC IMAGING FOR LOW BACK PAIN: ADVICE FOR HIGH-VALUE HEALTH CARE FROM THE AMERICAN COLLEGE OF PHYSICIANS. Annals Of Internal Medicine, [S.L.], v. 154, n. 3, p. 181-189, 1 fev. 2011. American College of Physicians. http://dx.doi.org/10.7326/0003-4819-154-3201102010-00008.

Disponível

em:

https://www.acpjournals.org/doi/full/10.7326/0003-4819-154-3-201102010-

00008?rfr_dat=cr_pub++0pubmed\&url_ver=Z39.88-

2003\&rfr_id=ori\%3Arid\%3Acrossref.org. Acesso em: 08 maio 2021.

DELLAROZA, Mara Solange Gomes et al. CARACTERIZAÇÃO DA DOR CRÔNICA E MÉTODOS ANALGÉSICOS UTILIZADOS POR IDOSOS DA COMUNIDADE. Revista da Associação Médica Brasileira, Londrina, Pr, v. 54, n. 1, p. 36-41, fev. 2008. Elsevier BV. http://dx.doi.org/10.1590/s0104-42302008000100018. Disponível

em:

https://www.scielo.br/j/ramb/a/d37LZLZ49k66xjWdjn7nG5v/?lang=pt. Acesso em: 11 mai. 2021.

FAGUNDES, F. R. C. et al. OREBRO QUESTIONNAIRE: SHORT AND LONG FORMS OF THE BRAZILIAN-PORTUGUESE VERSION. Quality of Life Research, [S.L.], v. 24, n. 11, p.2777-2788, jun. 2015. doi: 10.1007/s11136-0150998-3. Disponível em: https://link.springer.com/article/10.1007\%2Fs11136-0150998-3. Acesso em: 16 mai. 2021.

HALL, A.M. et al. DO NOT ROUTINELY OFFER IMAGING FOR UNCOMPLICATED LOW BACK PAIN. BMJ, [S.L.], v. 372, n. 291, fev. 2021. doi: https://doi.org/10.1136/bmj.n291. em: https://www.bmj.com/content/372/bmj.n291.full. Acesso em: 8 mai. 2021. 
HILL, Jonathan $\mathrm{C}$ et al. COMPARISON OF STRATIFIED PRIMARY CARE MANAGEMENT FOR LOW BACK PAIN WITH CURRENT BEST PRACTICE (START BACK): A RANDOMISED CONTROLLED TRIAL. The Lancet, [S.L.], v. 378, n. 9802, p. 1560-1571, out. 2011. Elsevier BV. http://dx.doi.org/10.1016/s01406736(11)60937-9. Disponível em: https://www.thelancet.com/journals/lancet/article/PIIS0140-6736(11)609379/fulltext\#. Acesso em: 16 mai. 2021.

HILL, Jonathan C. et al. A PRIMARY CARE BACK PAIN SCREENING TOOL: IDENTIFYING PATIENT SUBGROUPS FOR INITIAL TREATMENT. Arthritis \& Rheumatism, [S.L.], v. 59, n. 5, p. 632-641, 25 abr. 2008. Wiley. http://dx.doi.org/10.1002/art.23563. Disponível em: https://onlinelibrary.wiley.com/doi/full/10.1002/art.23563. Acesso em: 16 mai. 2021. HOY, Damian et al. A SYSTEMATIC REVIEW OF THE GLOBAL PREVALENCE OF LOW BACK PAIN. Arthritis \& Rheumatism, [S.L.], v. 64, n. 6, p. 2028-2037, jun. 2012. Wiley. http://dx.doi.org/10.1002/art.34347. Disponível em: https://onlinelibrary.wiley.com/doi/full/10.1002/art.34347. Acesso em: 08 mai. 2021. JACOBS, JC et al. OBSERVATIONAL STUDY OF THE DOWNSTREAM CONSEQUENCES OF INAPPROPRIATE MRI OF THE LUMBAR SPINE. J Gen Intern Med, [S.L.], v. 35, n. 12, p. 3605-3612, set. 2020. doi: 10.1007/s11606-02006181-7. Disponível em: https://link.springer.com/article/10.1007\%2Fs11606-02006181-7. Acesso em: 08 mai. 2021.

KRELING, Maria Clara Giorio Dutra; CRUZ, Diná de Almeira Lopes Monteiro da; PIMENTA, Cibele Andrucioli de Mattos. PREVALÊNCIA DE DOR CRÔNICA EM ADULTOS. Revista Brasileira de Enfermagem, [S.L.], v. 59, n. 4, p. 509-513, ago. 2006. FapUNIFESP (SciELO). http://dx.doi.org/10.1590/s003471672006000400007 . 
https://www.scielo.br/j/reben/a/JTJhBrgCTsMYjPhKxK6tbXN/?lang=pt. Acesso em: 11 mai. 2021.

LINTON, SJ; BOERSMA K. EARLY IDENTIFICATION OF PATIENTS AT RISK OF DEVELOPING A PERSISTENT BACK PROBLEM: THE PREDITIVE VALIDITY OF THE OREBRO MUSCULOSKELETAL PAIN QUESTIONNAIRE. The Clinical Journal of Pain, [S.L.], v.19, n.2, p. 80-86, mar-abr. 2003. doi: 10.1097 / $00002508-$ 200303000-00002.

https://journals.Iww.com/clinicalpain/Abstract/2003/03000/Early_Identification_of_Pa tients_at_Risk_of.2.aspx. Acesso em: 17 mai. 2021.

LINTON, Steven J.; SHAW, William S.. IMPACT OF PSYCHOLOGICAL FACTORS IN THE EXPERIENCE OF PAIN. Physical Therapy, [S.L.], v. 91, n. 5, p. 700-711, 1 maio 2011. Oxford University Press (OUP). http://dx.doi.org/10.2522/ptj.20100330. Disponível em: https://academic.oup.com/pti/article/91/5/700/2735743. Acesso em: 24 mai. 2021.

MAIN, Chris J.; FOSTER, Nadine; BUCHBINDER, Rachelle. HOW IMPORTANT ARE BACK PAIN BELIEFS AND EXPECTATIONS FOR SATISFACTORY RECOVERY FROM BACK PAIN? Best Practice \& Research Clinical Rheumatology, [S.L.], v. 24, n. 2, p. 205-217, abr. 2010. Elsevier BV. http://dx.doi.org/10.1016/j.berh.2009.12.012. Disponível em: https://pubmed.ncbi.nlm.nih.gov/20227642/. Acesso em: 16 mai. 2021.

NASCIMENTO, Paulo Roberto Carvalho do; COSTA, Leonardo Oliveira Pena. PREVALÊNCIA DA DOR LOMBAR NO BRASIL: UMA REVISÃO SISTEMÁTICA. Cadernos de Saúde Pública, [S.L.], v. 31, n. 6, p. 1141-1156, jun. 2015. FapUNIFESP (SciELO). http://dx.doi.org/10.1590/0102-311x00046114. Disponível em: 
Revista Multidisciplinar do Nordeste Mineiro, v.2,

$2021 / 02$

ISSN 2178-6925

https://www.scielo.br/j/csp/a/bXNZw98SnZTVK9CvDZt4TSg/?lang=pt. Acesso em: 07 mai. 2021.

O' Keeffe Mary et al. PSYCHOSOCIAL FACTORS IN LOW BACK PAIN: LETTING GO OF OUR MISCONCEPTIONS CAN HELP MANAGEMENT. British Journal of Sports Medicine, [S.L.], v. 53, n. 13, p. 793-794, ago. 2018. doi: 10.1136/bjsports2018-099816. Disponível em: https://bjsm.bmj.com/content/53/13/793.long. Acesso: 12 abr. 2021.

OLIVEIRA, CB et al. CLINICAL PRACTICE GUIDELINES FOR THE MANAGEMENT OF NON-SPECIFIC LOW BACK PAIN IN PRIMARY CARE: AN UPDATED OVERVIEW. European Spine Journal, [S.L.], v. 27, p. 2791-2803, jul. 2018. Disponível em: https://link.springer.com/article/10.1007/s00586-018-5673-2. Acesso em: 17 mai. 2021.

PILZ, Bruna et al. THE BRAZILIAN VERSION OF START BACK SCREENING TOOL - TRANSLATION, CROSS-CULTURAL ADAPTATION AND RELIABILITY. Brazilian Journal of Plysical Therapy, [S.L.], v.18, n.5, p. 453-461, set-out. 2014. https://doi.org/10.1590/bjpt-rbf.2014.0028.

Disponível

em: https://www.scielo.br/j/rbfis/a/XCg8m6v4TbDWnPBWR78bdLc/?lang=en. Acesso em: 30 abr. 2021.

RAJA, Srinivasa N. et al. THE REVISED INTERNATIONAL ASSOCIATION FOR THE STUDY OF PAIN DEFINITION OF PAIN: CONCEPTS, CHALLENGES, AND COMPROMISES, PAIN, [S.L.], v. 161, n. 9, p. 1976-1982, set, 2020. doi: 10.1097 / j.pain. 0000000000001939 .

Disponível

em: https://journals.Iww.com/pain/Abstract/2020/09000/The_revised_International_Asso ciation_for_the.6.aspx?context=LatestArticles. Acesso em: 11 mai. 2021. 
Revista Multidisciplinar do Nordeste Mineiro, v.2,

2021/02

ISSN 2178-6925

SOUZA, Juliana Barcellos de; BARROS, Carlos Marcelo de. CONSIDERATIONS ABOUT THE NEW CONCEPT OF PAIN. Brazilian Journal Of Pain, São Paulo, Sp, v. 3, n. 3, p. 294-294, set, 2020. GN1 Genesis Network. http://dx.doi.org/10.5935/2595-0118.20200190. Disponível em: https://www.scielo.br/j/brjp/a/r7Ff7DKVGD8B776KPRyMMPr/?lang=pt. Acesso em: 11 mai 2021.

TEGNER, Heidi et al. NEUROPHYSIOLOGICAL PAIN EDUCATION FOR PATIENTS WITH CHRONIC LOW BACK PAIN: A SYSTEMATIC REVIEW AND META-ANALYSIS. Clin J Pain, [S.L.], v. 34, n. 8, p. 778-786, ago. 2018. doi: 10.1097/AJP.0000000000000594. Disponível em: https://pubmed.ncbi.nlm.nih.gov/29443723/. Acesso em: 16 mai. 2021.

TELES, Alisson R. et al. PERSPECTIVE OF VALUE-BASED MANAGEMENT OF SPINAL DISORDERS IN BRAZIL. World Neurosurgery, [S.L.], v. 87, p. 346-354, mar. 2016. Elsevier BV. http://dx.doi.org/10.1016/j.wneu.2015.11.047. Disponível em:

https://www.sciencedirect.com/science/article/abs/pii/S1878875015016484?via\%3D ihub. Acesso em: 30 abr. 2021. 